\title{
Ant $\mathrm{i}$ - NXP2 aut oant $\mathrm{i}$ bodi es i $\mathrm{n}$ adul $\mathrm{t}$ pat $\mathrm{i}$ ent $\mathrm{s}$ wi th i di opat hi c i nf I ammat ory myopat hi es: Possi bl e associ at i on wi th nal i gnancy
}

\begin{tabular}{|c|c|}
\hline 著者 & $\begin{array}{l}\text { chi mur a Yuki, Nat sushi ta Takashi, Hamaguchi } \\
\text { Yasuhi to, Kaj i Kenzo, Hasegawa M nor u, Tani no } \\
\text { Yoshi nor i, I nokoshi Yayoi, Kawai Kazuhi ro, } \\
\text { Kanekur a Takur o, Habuchi Nar i a, I gar ashi } \\
\text { At suyuki, Sogame Ryosuke, Hashi not o Takashi, } \\
\text { Koga Tonmi ro, N shi no Ayako, I shi gur o Naoko, } \\
\text { Sugi mot o Naoki, Aoki Rui, Ando Nori ko, Abe } \\
\text { Test suya, Kanda Takashi, Kuwana Nasat aka, } \\
\text { Takehar a Kazuhi ko, Fuj i not o Nanabu }\end{array}$ \\
\hline $\begin{array}{l}\text { jour nal or } \\
\text { publ i cat } i \text { on } t i t l e\end{array}$ & Annal s of the Rheumat i c Di seases \\
\hline vol ume & 71 \\
\hline number & 5 \\
\hline page $r$ ange & $710-713$ \\
\hline year & 2012-05-01 \\
\hline URL & ht t p: //hdl . handl e. net /2297/31979 \\
\hline
\end{tabular}


Concise Report

\section{Anti-NXP2 autoantibodies in adult patients with idiopathic inflammatory myopathies: possible association with malignancy}

Yuki Ichimura, ${ }^{1}$ Takashi Matsushita, ${ }^{2}$ Yasuhito Hamaguchi, ${ }^{2}$ Kenzo Kaji, ${ }^{2}$ Minoru Hasegawa, ${ }^{2}$ Yoshinori Tanino, ${ }^{3}$ Yayoi Inokoshi, ${ }^{3}$ Kazuhiro Kawai, ${ }^{4}$ Takuro Kanekura, ${ }^{4}$ Maria Habuchi, ${ }^{5}$ Atsuyuki Igarashi, ${ }^{5}$ Ryosuke Sogame, ${ }^{6}$ Takashi Hashimoto, ${ }^{6}$ Tomohiro Koga, ${ }^{7}$ Ayako Nishino, ${ }^{7}$ Naoko Ishiguro, ${ }^{8}$ Naoki Sugimoto, ${ }^{9}$ Rui Aoki, ${ }^{10}$ Noriko Ando, ${ }^{10}$ Testsuya Abe, ${ }^{11}$ Takashi Kanda, ${ }^{11}$ Masataka Kuwana, ${ }^{12}$ Kazuhiko Takehara, ${ }^{2}$ Manabu Fujimoto $^{2}$

${ }^{1}$ School of Medicine and ${ }^{2}$ Department of Dermatology, Graduate School of Medical Science, Kanazawa University, Kanazawa; ${ }^{3}$ Department of Pulmonary Medicine, Fukushima Medical University School of Medicine, Fukushima; ${ }^{4}$ Department of Dermatology, Kagoshima University Graduate School of Medical and Dental Sciences, Kagoshima; ${ }^{5}$ Division of Dermatology, Kanto Medical Center NTT EC, Tokyo; ${ }^{6}$ Department of Dermatology, Kurume University School of Medicine, Kurume; ${ }^{7}$ Unit of Translational Medicine, Department of Immunology and Rheumatology, Graduate School of Biomedical Sciences, Nagasaki University, Nagasaki; ${ }^{8}$ Department of Dermatology and ${ }^{9}$ Institute of Rheumatology, Tokyo Women's Medical University, Tokyo; ${ }^{10}$ Department of Dermatology, Faculty of Medicine, University of Yamanashi, Yamanashi; 11 Department of Neurology and Clinical Neuroscience, Yamaguchi University Graduate School of Medicine, Ube; ${ }^{12}$ Division of Rheumatology, Department of Internal Medicine, Keio University School of Medicine, Tokyo, Japan 


\section{Correspondence to}

Manabu Fujimoto, M.D., Department of Dermatology, Kanazawa University Graduate

School of Medical Science, 13-1 Takaramachi, Kanazawa, Ishikawa, 920-8641, Japan.

E-mail: fujimoto-m@umin.ac.jp. Tel.: +81-76-265-2341. Fax: +81-76-234-4270. 


\section{ABSTRACT}

Objectives. Myositis-specific autoantibodies (MSAs) are useful tools in identifying clinically homogenous subsets and predicting prognosis of patients with idiopathic inflammatory myopathies (IIM) including polymyositis (PM) and dermatomyositis (DM). Recent studies have revealed that anti-NXP2 antibody (Ab) is a major MSA in juvenile dermatomyositis (JDM). In this study, we evaluated the frequencies and clinical associations of anti-NXP2 Ab in adult IIM patients.

Methods. Clinical data and sera were collected from 507 adult Japanese patients with IIM (445 adult DM, and 62 adult PM). As disease controls, 11 with JDM, 108 with systemic lupus erythematosus, 433 with systemic sclerosis, and 124 with idiopathic pulmonary fibrosis were assessed. Sera were examined for anti-NXP2 Ab by immunoprecipitation and Western blotting using polyclonal anti-NXP2 Ab.

Results. Seven patients (1.6\%) with adult DM and 1 (1.6\%) with adult PM were positive for anti-NXP2 Ab. Except for 2 patients with JDM, no patients as disease controls were positive for this autoAb. Among 8 adult IIM patients, 3 had internal malignancies within 3 years of diagnosis of IIM. Another DM patient also had a metastatic cancer at the diagnosis. All of the carcinomas were advanced stage (stage IIIb-IV).

Conclusions. While less common than in juvenile IIM, anti-NXP2 Ab was found in adult IIM. Anti-NXP2 Ab may be associated with adult IIM with malignancy.

Key words; dermatomyositis, polymyositis, myositis-specific autoantibody, NXP2, malignancy 


\section{INTRODUCTION}

Idiopathic inflammatory myopathies (IIM), including polymyositis (PM) and dermatomyositis (DM), are characterized by chronic inflammation of skeletal muscles and/or skin eruptions [1]. Although the etiology remains unclear, they are considered as autoimmune diseases. The presence of disease-specific autoantibodies (autoAbs), known as myositis-specific autoAbs (MSAs), is a prominent feature. Moreover, MSAs are strongly associated with distinct clinical phenotypes and thus classify patients into groups with more homogenous clinical features [2-4]. These MSAs include antibodies (Abs) to aminoacyl-tRNA synthetases (ARS), the Mi-2 protein, the signal-recognition particle (SRP), transcriptional intermediary factor-1 (TIF1; anti-155/140 Ab) [5-7], and melanoma differentiation-associated gene-5 (MDA5; anti-CADM140 Ab) [8].

Oddis and colleagues first described anti-MJ Ab in a US cohort of juvenile DM (JDM) [9], and Targoff et al. subsequently identified that the antigen of anti-MJ Ab is nuclear matrix protein NXP2 (MORC3) [10]. Gunawardena et al. and Espada et al. have demonstrated that anti-NXP2 Ab is among the most common MSAs in JDM [11, 12]. In this study, we evaluated the frequencies and clinical associations of anti-NXP2 Ab in adult IIM patients. 


\section{PATIENTS AND METHODS}

\section{Patients}

Serum samples were obtained from 507 consecutive Japanese adult patients with IIM, 445 with DM and 62 with PM, who had been followed up in the Department of Dermatology, Kanazawa University Hospital and collaborating medical centers from 2003 to 2010. All PM patients and 365 DM patients fulfilled Bohan and Peter's criteria $[13,14]$. The remaining 80 did not, but fulfilled Sontheimer's criteria of clinically amyopathic DM (CADM) [15]. Among DM patients, As disease controls, 11 with JDM. 108 with systemic lupus erythematosus, 433 with systemic sclerosis, and 124 with idiopathic pulmonary fibrosis were assessed. Clinical information was collected retrospectively by reviewing their clinical medical charts. The protocol was approved by Kanazawa University Graduate School of Medical Science.

\section{Immunoprecipitation and Western blotting}

Ten microliters of serum was incubated with $2 \mathrm{mg}$ of protein A-Sepharose beads (Amersham Biosciences, Piscataway, NJ, USA) in immunoprecipitation buffer (10 mM Tris-HCl, pH 8.0, $50 \mathrm{mM} \mathrm{NaCl}, 0.1 \%$ Nonidet P-40) for 2 hours. Beads were then mixed with ${ }^{35}$ S-labeled or unlabeled K562 cell extracts derived from $10^{7}$ cells and rotated at $4{ }^{\circ} \mathrm{C}$ for 2 hours. After five washes, precipitated proteins were fractionated by SDS-polyacrylamide gel electrophoresis (SDS-PAGE), followed by autoradiography or Western blotting.

For Western blotting, proteins were transferred onto a nitrocellulose membrane. After blocking, the membrane was incubated with mouse polyclonal anti-human NXP2 Ab (Abcam, Cambridge, UK), and then with horseradish peroxidase-conjugated goat anti-mouse IgG Ab (Thermo Scientific, Rockford, IL, USA). The membrane was developed using an enhanced chemiluminescence kit (Thermo Scientific). 


\section{Statistic analysis}

Fisher's exact probability tests were used for comparison of frequencies. Bonferroni's test was examined for multiple comparisons of values following normal distribution. The values that were not normally distributed were evaluated by Mann-Whitney U test. $\mathrm{P}$ values less than 0.05 were considered significant. 


\section{RESULTS}

\section{Detection of anti-NXP2 Abs}

Sera from 507 adult Japanese IIM patients were screened for anti-NXP2 Ab. From K562 cell extracts, 8 sera precipitated a $140-\mathrm{kDa}$ protein, which was different from other known autoantigens (Figure 1A). Additionally, 2 JDM patients were positive for this $140-\mathrm{kDa}$ protein. No sera from other diseases precipitated this $140-\mathrm{kDa}$ protein. On SDS-PAGE, the 140-kDa band was slightly higher than the 140-kDa band of anti-TIF1 $\mathrm{Ab}$, and was not accompanied by a $155-\mathrm{kDa}$ band (Figure 1A). While this 140-kDa protein migrated closely to MDA5, these sera were negative for anti-MDA5 Ab by an enzyme-linked immunosorbent assay using recombinant MDA5 protein as antigen (data not shown). These sera were confirmed to react with NXP2 since precipitated proteins were recognized by polyclonal Abs to NXP2 in Western blotting (Figure 1B). They also reacted with recombinant human NXP2 protein by Western blotting (online supplementary Figure S1). These sera were negative for other MSAs, and did not have other known autoAbs, except for patient B, who was positive for anti-SS-A/Ro and SS-B/La Abs. In indirect immunofluorescence, 5 showed negative staining, while 3 stained nuclei in speckled pattern at the maximum dilution of x80.

\section{Clinical and laboratory profiles of IIM patients with anti-NXP2 Abs}

Among 507 adult IIM patients, 8 patients were positive for anti-NXP2 Ab: 7 (1.6\%) in 445 adult DM patients and 1 (1.6\%) in 62 adult PM patients. All patients with anti-NXP2 Ab showed strong muscle weakness and high elevation of serum CK levels. Remarkably, internal malignancies found within 3 years of the diagnosis of IIM were present in 37.5\% (3/8) (Table 2). All these patients had advanced disease (satage IIIb-IV). Additionally, in a patient (C), prostate cancer was found 42 months before, and it was metastatic when the diagnosis of DM was made. However, he was excluded from the statistical analysis since he did not meet the above criteria. ILD was not found. 
During the follow-up period varying from 2 to 61 months, 7 of 8 patients, except for patient $\mathrm{H}$, were treated with systemic corticosteroid therapy. Patient A also received methotrexate, and patient $\mathrm{D}$ also underwent intravenous Ig therapy. While the response to the treatment was favorable in all patients, 2 (C and $\mathrm{G}$ ) died of malignancy.

\section{Comparison with other MSAs}

In addition to 7 patients positive for anti-NXP2 Ab, adult DM patients in this study included 74 patients with anti-TIF1 Ab and 15 patients with anti-Mi-2 Ab. They also included 51 with anti-MDA5 Ab, 26 with anti-PL-7 Ab, 18 with anti-Jo-1 Ab, and 8 with anti-PL-12 Ab. Among them, since anti-TIF1 Ab and anti-Mi-2 Ab are specifically associated with DM, the clinical features of patients with anti-NXP2 Ab were compared with those with anti-TIF1 Ab and anti-Mi-2 Ab (Table 2).

Anti-NXP2 Ab was predominantly found in male patients. Although Gottron's sign was slightly less frequent, there was no significant difference for the frequency of each cutaneous manifestation. The frequency of fever was significant higher than that in anti-TIF1-positive DM patients. The highest CK level was similar to that of anti-Mi-2 Ab-positive DM, and significant higher than that of anti-TIF1 Ab. The frequency of internal malignancy in those with anti-NXP2 Ab was lower than that in anti-TIF1 Ab, and higher than that in anti-Mi-2 Ab, although the differences were not statistically significant. 


\section{DISCUSSION}

Recently, two studies demonstrated that anti-NXP2 Ab was detected in 23\% and 25\% of JDM patients in UK and Argentina, respectively [11, 12]. While the number of patients was small, anti-NXP2 Ab was detected in 18\% in the control JDM population in this study. Therefore, anti-NXP2 Ab is likely to be a major MSA in JDM across racial groups as well as anti-TIF1 Ab, which is detected at 23-29\% in JDM [16]. In addition to JDM, Espada et al. reported that 2 (28\%) of 7 patients with juvenile PM were also positive for anti-NXP2 Ab [12]. In this study, we identified 8 anti-NXP2 Ab-positive adult patients in a Japanese cohort of IIM. The frequencies were 1.6\% in both adult DM and adult PM. Therefore, while the population sizes of DM and PM are substantially different, anti-NXP2 Ab has been found at similar frequencies both in juvenile and adult IIM [12]. By contrast, in a UK cohort, Betteridge et al. preliminarily reported that anti-NXP2 Ab was detected in 13 (5\%) DM patients but not in PM patients [17].

Intriguingly, $37.5 \%$ of adult IIM patients positive for anti-NXP2 Ab had malignancy which was found within 3 years in addition to a patient with metastatic prostate cancer found 42 months before. Moreover, all of these carcinomas were at an advanced stage. Anti-TIF1 Ab also has a strong association with internal malignancy in adult DM, as it is found in $50-70 \%$ of cancer-associated DM patients $[5,6,18]$. Therefore, while anti-NXP2 Ab may not be restricted to DM, anti-TIF1 Ab and anti-NXP2 Ab may have a shared property in that they represent two clinical subsets of cancer-associated adult DM and JDM. However, in contrast to our study, Betteridge et al. reported that anti-NXP2 Ab was not associated with malignancy but with interstitial lung disease [17]. This may be due to ethnical differences. It may have also resulted from different distribution of patients between Dermatology and Rheumatology clinics. Since both studies had relatively small numbers of patients, more studies are needed to evaluate more precise clinical relevance of anti-NXP2 Ab in IIM patients. 
Among adult DM patients, anti-NXP2 Ab appeared to have strong muscle involvement, while we could not find any particular cutaneous manifestations related to anti-NXP2 Ab. Unlike anti-NXP2-positive JDM [11], no adult DM patients with anti-NXP2 Ab exhibited cutaneous calcinosis. This may be due to the relatively low incidence of calcinosis in adult DM compared with that in JDM.

In summary, this study described that anti-NXP2 Ab was found in adult IIM patients, and suggesting a possibility that anti-NXP2 Ab is correlated with cancer-associated myositis. NXP2 is involved in the activation and localization of a tumor suppressor gene, p53 [19]. TIF1 proteins also have functional relationship with p53 [20]. Since these two autoAbs may share similar clinical characteristics, especially the association with cancer, they may develop during anti-tumor immune responses. 
Table 1. Clinical and laboratory profile of adult patients with dermatomyositis (DM) or polymyositis (PM) positive for anti-NXP2 Ab*

\begin{tabular}{|c|c|c|c|c|c|c|c|c|}
\hline Patient & A & B & $\mathrm{C}$ & $\mathrm{D}$ & $\mathrm{E}$ & $\mathrm{F}$ & G & $\mathrm{H}$ \\
\hline Diagnosis & $\mathrm{DM}$ & $\mathrm{DM}$ & $\mathrm{DM}$ & $\mathrm{DM}$ & $\mathrm{DM}$ & $\mathrm{DM}$ & $\mathrm{PM}$ & $\mathrm{DM}$ \\
\hline Duration (mo) & 11 & 21 & 10 & 8 & 23 & 4 & 2 & 7 \\
\hline Gottron's sign & + & - & + & + & - & - & - & + \\
\hline Perionychia erythema & - & - & + & + & + & + & - & - \\
\hline Nailfold punctuated hemorrhage & - & - & + & - & + & + & - & + \\
\hline erythema of extensor extremities & - & + & + & + & + & + & - & - \\
\hline Calcinosis & - & - & - & - & - & - & - & - \\
\hline Blistering & - & - & - & - & - & - & - & - \\
\hline Ulceration & - & - & - & - & + & - & - & - \\
\hline Muscle weakness & + & + & + & + & + & + & + & + \\
\hline
\end{tabular}




\begin{tabular}{|c|c|c|c|c|c|c|c|c|}
\hline Raynaud's phenomenon & - & - & - & - & - & - & - & - \\
\hline Fever & - & - & + & - & + & + & + & + \\
\hline Arthralgia & - & + & - & - & - & - & - & - \\
\hline Elevation of CK & + & + & + & + & + & + & + & + \\
\hline Highest CK level (IU/l) & 3857 & 1877 & 4927 & 1539 & 17140 & 26685 & 3713 & 3722 \\
\hline IIF titer & $<40$ & 80 & $<40$ & 40 & $<40$ & $<40$ & 80 & $<40$ \\
\hline IIF staining pattern & - & Sp & - & Sp & - & - & Sp & - \\
\hline ILD & - & - & - & - & - & - & - & - \\
\hline Malignancy & - & - & + & + & - & - & + & + \\
\hline Origin & - & - & Prostate & Pancreas & - & - & Gallbladder & Lung \\
\hline Histology & - & - & $\mathrm{AC}$ & ND & - & - & $\mathrm{AC}$ & SCC \\
\hline Stage & - & - & IV & $\mathrm{IVb}$ & - & - & $\mathrm{IVb}$ & IIIb \\
\hline Period of diagnosis** & - & - & 42 mo before & 6 mo after & - & - & Simultaneous & 3 mo before \\
\hline
\end{tabular}

*CADM, clinically amyopathic DM; mo, month; CK, creatine kinase; IIF, indirect immunofluorescence; Sp, speckled; ILD, interstitial lung disease; AC, adenocarcinoma; SSC, squamous cell carcinoma; ND, not done. ** Period of diagnosis indicates when the diagnosis of malignancy was made before or after the onset of IIM. 
Table 2. Comparison of clinical and laboratory profile of adult dermatomyositis patients with anti-NXP2 Ab, anti-TIF1 Ab, and anti-Mi-2 Ab*

\begin{tabular}{|c|c|c|c|c|c|}
\hline & \multirow[b]{2}{*}{ anti-NXP2-positive } & \multirow[b]{2}{*}{ anti-TIF1-positive } & \multirow[b]{2}{*}{ anti-Mi-2-positive } & \multicolumn{2}{|c|}{ P-Values } \\
\hline & & & & vs. anti-TIF1 & vs. anti-Mi-2 \\
\hline Number & 7 & 74 & 15 & & \\
\hline Age at onset, mean (range) & $57(23-68)$ & $59(27-89)$ & $50(16-67)$ & N.S. & N.S. \\
\hline Sex (male:female) & $6: 1$ & 24: 34 & 8: 7 & 0.033 & N.S. \\
\hline \multicolumn{6}{|l|}{ Skin eruptions } \\
\hline Heliotrope rash & 71 & 62 & 60 & N.S. & N.S. \\
\hline Gottron's sign & 57 & 81 & 87 & N.S. & N.S. \\
\hline Perionychia erythema & 57 & 57 & 60 & N.S. & N.S. \\
\hline Nailfold punctuated hemorrhage & 57 & 40 & 73 & N.S. & N.S. \\
\hline Trunk erythema & 57 & 62 & 53 & N.S. & N.S. \\
\hline Calcinosis & 0 & 2 & 7 & N.S. & N.S. \\
\hline Ulceration & 14 & 7 & 0 & N.S. & N.S. \\
\hline \multicolumn{6}{|l|}{ Clinical features } \\
\hline Muscle weakness & 100 & 69 & 93 & N.S. & N.S. \\
\hline Raynaud phenomenon & 0 & 9 & 0 & N.S. & N.S. \\
\hline Arthritis & 14 & 3 & 7 & N.S. & N.S. \\
\hline
\end{tabular}


Fever

57

16

20

0.025

N.S.

Organ involvement

Interstitial lung disease

Internal malignancy within 3 years

0

29

Laboratory findings

Elevated CK, \%

100

63

Highest CK level, IU/l, mean (range) 3857 (1539-26685)

$425(40-8670)$
13

7

100
N.S.

N.S.

N.S.

N.S.

3934 (401-10000)

$<0.001$

N.S.

* Unless noted, otherwise, values are percentages. 


\section{Acknowledgements}

We thank Ms. Masako Matsubara, Ms. Tomoko Hayashi, and Ms. Natsuho Yoshifuji for technical assistance.

\section{Funding}

This work was supported by a grant of Research on Intractable Diseases from the Ministry of Health, Labour and Welfare of Japan.

\section{Competing interests}

The authors declare no conflict of interest.

\section{Ethic approval}

This study was conducted with the approval of Kanazawa University Graduate School of Medical Science and Kanazawa University Hospital. 


\section{REFERENCES}

1. Zong $\mathrm{M}$, Lundberg IE. Pathogenesis, classification and treatment of inflammatory myopathies. Nat Rev Rheumatol. 2011; 7:297-306.

2. Targoff IN. Myositis specific autoantibodies. Curr Rheumatol Rep. 2006; 8:196-203.

3. Gunawardena H, Betteridge ZE, McHugh NJ. Myositis-specific autoantibodies: their clinical and pathogenic significance in disease expression. Rheumatology (Oxford). 2009; 48:607-612.

4. Hamaguchi Y, Kuwana M, Hoshino K, et al. Clinical Correlations With Dermatomyositis-Specific Autoantibodies in Adult Japanese Patients With Dermatomyositis: A Multicenter Cross-sectional Study. Arch Dermatol. 2011; 147:391-398.

5. Targoff IN, Mamyrova G, Trieu EP, et al. A novel autoantibody to a 155-kd protein is associated with dermatomyositis. Arthritis Rheum. 2006; 54:3682-3689.

6. Kaji K, Fujimoto M, Hasegawa M, et al. Identification of a novel autoantibody reactive with 155 and $140 \mathrm{kDa}$ nuclear proteins in patients with dermatomyositis: an association with malignancy. Rheumatology (Oxford). 2007; 46:25-28.

7. Fujimoto M, Hamaguchi Y, Kaji K, et al. Myositis-specific anti-155/140 autoantibodies target transcriptional intermediary factor 1 family proteins. Arthritis Rheum. in press.

8. Sato S, Hoshino K, Satoh $\mathrm{T}$, et al. RNA helicase encoded by melanoma differentiation-associated gene 5 is a major autoantigen in patients with clinically amyopathic dermatomyositis: Association with rapidly progressive interstitial lung disease. Arthritis Rheum. 2009; 60:2193-2200.

9. Oddis CV, Fertig N, Goel A, et al. Clinical and serological characterization of the anti-MJ antibody in childhood myositis [abstract]. Arthritis Rheum. 1997; 40:S139. 
10. Targoff IN, Trieu EP, Levy-Neto M, et al. Sera with autoantibodies to the MJ antigen react with NXP2 [abstract]. Arthritis Rheum. 2007; 56:S787.

11. Gunawardena H, Wedderburn LR, Chinoy H, et al. Autoantibodies to a 140-kd protein in juvenile dermatomyositis are associated with calcinosis. Arthritis Rheum. 2009; 60:1807-1814.

12. Espada G, Maldonado Cocco JA, Fertig N, et al. Clinical and serologic characterization of an Argentine pediatric myositis cohort: identification of a novel autoantibody (anti-MJ) to a 142-kDa protein. J Rheumatol. 2009; 36:2547-2551.

13. Bohan A, Peter JB. Polymyositis and dermatomyositis (second of two parts). N Engl J Med. 1975; 292:403-407.

14. Bohan A, Peter JB. Polymyositis and dermatomyositis (first of two parts). N Engl J Med. 1975; 292:344-347.

15. Sontheimer RD. Would a new name hasten the acceptance of amyopathic dermatomyositis (dermatomyositis sine myositis) as a distinctive subset within the idiopathic inflammatory dermatomyopathies spectrum of clinical illness? J Am Acad Dermatol. 2002; 46:626-636.

16. Gunawardena H, Wedderburn LR, North J, et al. Clinical associations of autoantibodies to a $\mathrm{p} 155 / 140 \mathrm{kDa}$ doublet protein in juvenile dermatomyositis. Rheumatology (Oxford). 2008; 47:324-328.

17. Betteridge Z, Gunawardena H, Chinoy $\mathrm{H}$, et al. Clinical associations of anti-p140 autoantibodies in adult myositis. [abstract]. Ann Rheum Dis. 2010; 69 (suppl3):127.

18. Chinoy H, Fertig $\mathrm{N}$, Oddis $\mathrm{CV}$, et al. The diagnostic utility of myositis autoantibody testing for predicting the risk of cancer-associated myositis. Ann Rheum Dis. 2007; 66:1345-1349.

19. Takahashi K, Yoshida N, Murakami N, et al. Dynamic regulation of p53 
subnuclear localization and senescence by MORC3. Mol Biol Cell. 2007; 18:1701-1709.

20. Jain AK, Barton MC. Regulation of p53: TRIM24 enters the RING. Cell Cycle. 2009; 8:3668-3674. 


\section{Figure Legends}

Figure 1. Detection of anti-NXP2 Ab. A, Immunoprecipitant from ${ }^{35}$ S-labeled K562 cell extract using serum samples were subjected to 7\% SDS-PAGE. Lanes A-H correspond to anti-NXP2-positive patients shown in Table 1. As controls, the prototype sera positive for anti-TIF1 Ab and anti-MDA5 Ab and normal control serum were also examined. Molecular weights (MW) are shown on the left. The position of the 140-kDa NXP2 protein is indicated by an arrow. B, K562 cell extracts were immunoprecipitated with anti-NXP2-positive sera (patients A-H), anti-MDA5-positive sera, anti-TIF1-positive sera, or normal control sera, and were subjected to SDS-PAGE, electro-transferred onto nitrocellulose membranes, and probed with polyclonal anti-NXP2 Ab. The molecular weight of NXP2 (140 kDa) is indicated by an arrow. 

A
Anti-MDA5 Anti-TIF1 MW A B C D E F G H $\downarrow \downarrow \downarrow$

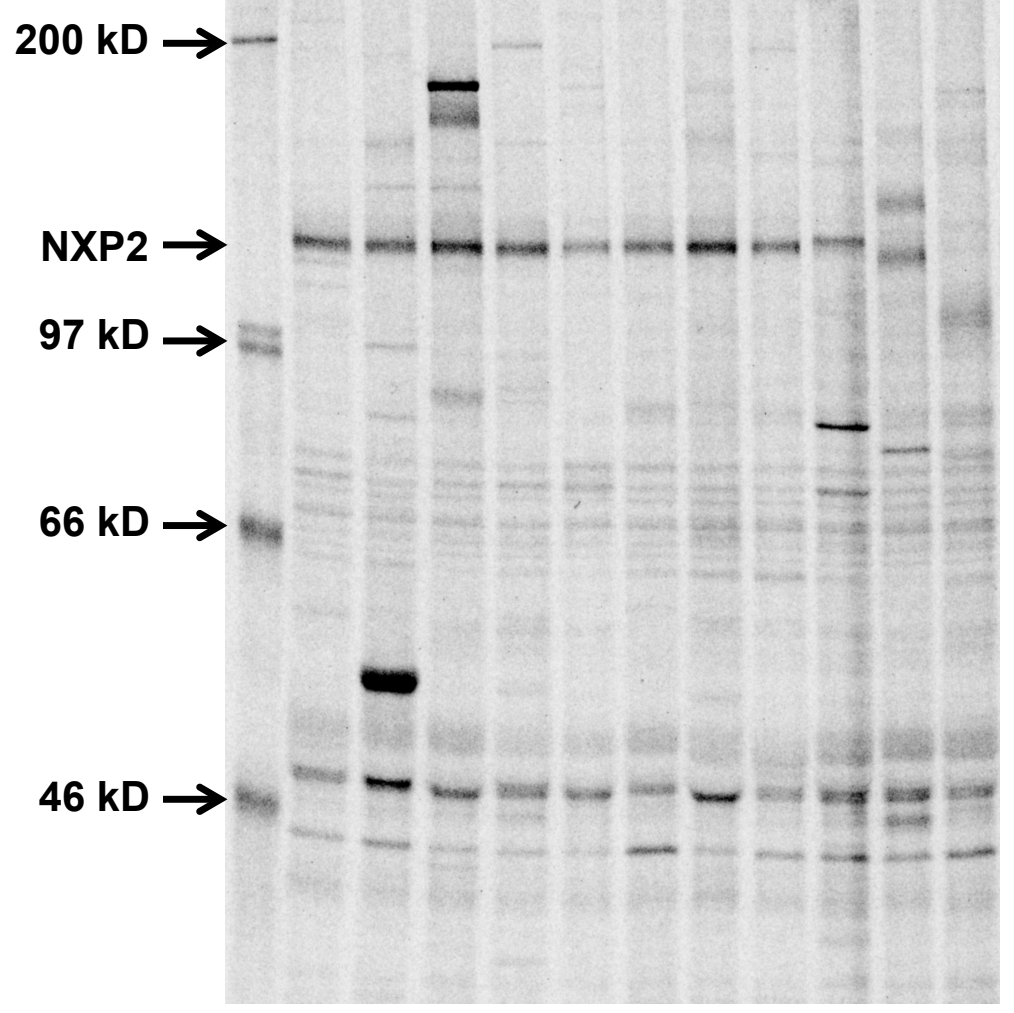

B

\begin{tabular}{|c|c|c|}
\hline \multicolumn{2}{|c|}{ Anti-MDA5 } & \multirow{2}{*}{$\begin{array}{l}\text { Anti-TIF1 } \\
\text { Normal control }\end{array}$} \\
\hline & Normal cc & \\
\hline Anti-NXP2 & Anti-NXP2 & \\
\hline
\end{tabular}

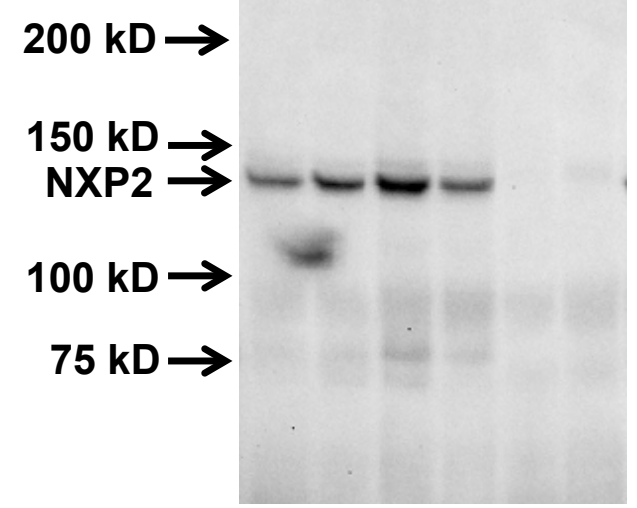

Figure 1 


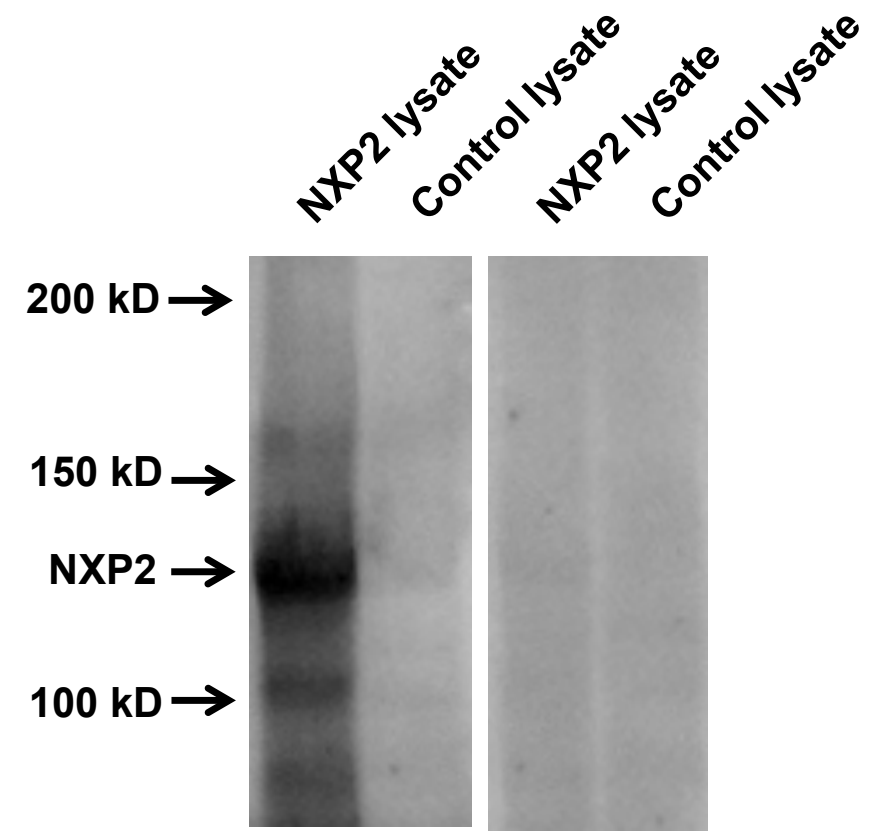

Anti-NXP2 sera Control sera 